HIP-2010-17/TH

BI-TP 2010/18

\title{
Scale-dependence of Non-Gaussianity in the Curvaton Model
}

\author{
Christian T. Byrnes ${ }^{1}$, Kari Enqvist ${ }^{2,3}$ and Tomo Takahashi ${ }^{4}$ \\ ${ }^{1}$ Fakultät für Physik, Universität Bielefeld, Postfach 100131, 33501 Bielefeld, Germany \\ ${ }^{2}$ Helsinki Institute of Physics, University of Helsinki, PO Box 64, FIN-00014, Finland \\ 3 Department of Physical Science, University of Helsinki, \\ PO Box 64, FIN-00014, Finland \\ 4 Department of Physics, Saga University, Saga 840-8502, Japan
}

\begin{abstract}
We investigate the scale-dependence of $f_{\mathrm{NL}}$ in the self-interacting curvaton model. We show that the scale-dependence, encoded in the spectral index $n_{f_{\mathrm{NL}}}$, can be observable by future cosmic microwave background observations, such as CMBpol, in a significant part of the parameter space of the model. We point out that together with information about the trispectrum $g_{\mathrm{NL}}$, the self-interacting curvaton model parameters could be completely fixed by observations. We also discuss the scaledependence of $g_{\mathrm{NL}}$ and its implications for the curvaton model, arguing that it could provide a complementary probe in cases where the theoretical value of $n_{f_{\mathrm{NL}}}$ is below observational sensitivity.
\end{abstract}




\section{Introduction}

It is now widely recognized that non-Gaussianity is a useful tool to distinguish between various models for the primordial perturbation. Although the power spectrum of the CMB temperature fluctuations are well constrained by current observations such as WMAP [1], at linear order many mechanisms can give rise to almost the same spectrum of fluctuations. Thus, to differentiate between models, one needs to go beyond the linear order. For example, the standard slow-roll inflation models predict an almost Gaussian primordial perturbation, while other mechanism such as the curvaton [2 4, yield almost identical power spectrum but can have a significant non-Gaussian component.

The deviations from the purely Gaussian perturbation are usually characterized by some non-linearity parameters, such as $f_{\mathrm{NL}}$, related to the 3 -point function, and $\tau_{\mathrm{NL}}$ and $g_{\mathrm{NL}}$, which are related to the 4 -point function. The present observational constraint on $f_{\mathrm{NL}}$ is $-10<f_{\mathrm{NL}}<74$ (95\%C.L.) [1]\#1. If taken at face value, the current limit might actually suggest that $f_{\mathrm{NL}}$ deviates from zero (although it is still consistent with zero); this might spell trouble for the standard single-field slow-roll inflation models, which typically give $f_{\mathrm{NL}}$ which is proportional to the small slow-roll parameters, or at most $f_{\mathrm{NL}} \sim \mathcal{O}(1)[10]$. If this suggestion turns out to be confirmed by Planck, the curvaton models would be natural candidates for the primordial perturbation.

Indeed, non-Gaussianity in the curvaton model has been the subject of extensive study [11 29]. However, in the majority of these studies the curvaton potential is assumed to be of the quadratic form. Since in order to be able to decay, the curvaton has to couple to some other fields, it then follows that through loop corrections there will also appear some effective curvaton self-couplings, even if they were absent at tree level. Moreover, it has been pointed out that even small deviations from the quadratic potential may have significant effects for the primordial perturbation if due to the curvaton [12, 15, 21, 28, In particular, it has been shown that for non-quadratic curvaton potentials the predictions for $f_{\mathrm{NL}}$ and $g_{\mathrm{NL}}$ can be quite different as compared with the purely quadratic case [15, 21. In the presence of non-linearities, it is also possible that the non-Gaussianity of the perturbation can be revealed by $g_{\mathrm{NL}}$ (or the 4-point function) rather than by $f_{\mathrm{NL}}$, which by virtue of the non-linearites can remain very small for certain choices of the parameters [21].

Another point worth stressing is that the well-known relation $f_{\mathrm{NL}} \sim 1 / r_{\text {dec }}$ for the curvaton model, where $r_{\text {dec }}$ is related to the curvaton fraction of the total energy density at the time of curvaton decay, is not generic and strictly speaking only true for the quadratic potential [28]. Thus a general, self-interacting curvaton model is a source for new observational signatures. However, self-interaction means also more free parameters. Hence it would be desirable to have more observational quantities beyond $f_{\mathrm{NL}}$ and $g_{\mathrm{NL}}$ that would help to constrain the models.

Indeed, recently it has been pointed out that the scale-dependence of $f_{\mathrm{NL}}$ could be measured by future observations [30]. Theoretical aspects of the scale-dependence have been investigated in [31 34]. We note that the scale dependence of equilateral type non-

${ }^{\# 1}$ For the constraints on $\tau_{\mathrm{NL}}$ and $g_{\mathrm{NL}}$, see [5] 7] and for forecasts on future constraints see [8, 9]. 
Gaussianity is also of interest [36 39]. Here we study the scale-dependence of the nonGaussianity parameters in a self-interacting curvaton model. We show that, in some cases, the scale-dependence of $f_{\mathrm{NL}}$ can be large enough to be detected in future observations. We also demonstrate that by using the combined information about $f_{\mathrm{NL}}$ and $g_{\mathrm{NL}}$, we may essentially fix the curvaton model parameters.

The organization of this paper is as follows. In the next section, we summarize the formalism for the study of the scale-dependence of $f_{\mathrm{NL}}$, which manifests itself in the spectral index $n_{f_{\mathrm{NL}}}$, in a general curvaton model. Then in Section 3, we closely look at $n_{f_{\mathrm{NL}}}$ in various cases and discuss its detectability in future observations. We also discuss the implications for $g_{\mathrm{NL}}$, in particular focusing on the complementary nature of the combined information. The final section is devoted to the summary and a discussion about the implications of the results.

\section{Formalism and Definitions}

For the purpose of discussing the scale-dependence of non-Gaussianity parameters in the curvaton model, let us assume that the potential for the curvaton $\sigma$ can be written as

$$
V(\sigma)=\frac{1}{2} m_{\sigma}^{2} \sigma^{2}+\lambda m_{\sigma}^{4}\left(\frac{\sigma}{m_{\sigma}}\right)^{p}
$$

where $m_{\sigma}$ is the mass of the curvaton, and $\lambda$ and $p$ represent the strength and the power of the self-coupling, respectively. To characterize the relative size of the self-interaction term in the potential, we define the parameter $s$ as follows:

$$
s \equiv 2 \lambda\left(\frac{\sigma_{*}}{m_{\sigma}}\right)^{p-2}
$$

where the subscript $*$ represents that the quantity is evaluated at the time the scale leaves the horizon. Thus $s$ is the ratio of two terms in Eq. (1) at the horizon exit. Once the curvaton begins to oscillate, the energy density of the curvaton can soon be well described by the purely quadratic case (i.e., the "nearly" quadratic case discussed in [15, 21]), the curvature perturbation up to the third order, adopting sudden decay approximation, can be written as \#2

$$
\begin{aligned}
\zeta= & \frac{2}{3} r_{\mathrm{dec}} \frac{\sigma_{\mathrm{osc}}^{\prime}}{\sigma_{\mathrm{osc}}} \delta \sigma_{*}+\frac{1}{9}\left[3 r_{\mathrm{dec}}\left(1+\frac{\sigma_{\mathrm{osc}} \sigma_{\mathrm{osc}}^{\prime \prime}}{\sigma_{\mathrm{osc}}^{\prime 2}}\right)-4 r_{\mathrm{dec}}^{2}-2 r_{\mathrm{dec}}^{3}\right]\left(\frac{\sigma_{\mathrm{osc}}^{\prime}}{\sigma_{\mathrm{osc}}}\right)^{2}\left(\delta \sigma_{*}\right)^{2} \\
& +\frac{4}{81}\left[\frac{9 r_{\mathrm{dec}}}{4}\left(\frac{\sigma_{\mathrm{osc}}^{2} \sigma_{\mathrm{osc}}^{\prime \prime \prime}}{\sigma_{\mathrm{osc}}^{\prime 3}}+3 \frac{\sigma_{\mathrm{osc}} \sigma_{\mathrm{osc}}^{\prime \prime}}{\sigma_{\mathrm{osc}}^{\prime 2}}\right)-9 r_{\mathrm{dec}}^{2}\left(1+\frac{\sigma_{\mathrm{osc}} \sigma_{\mathrm{osc}}^{\prime \prime}}{\sigma_{\mathrm{osc}}^{\prime 2}}\right)\right. \\
& \left.+\frac{r_{\mathrm{dec}}^{3}}{2}\left(1-9 \frac{\sigma_{\mathrm{osc}} \sigma_{\mathrm{osc}}^{\prime \prime}}{\sigma_{\mathrm{osc}}^{\prime 2}}\right)+10 r_{\mathrm{dec}}^{4}+3 r_{\mathrm{dec}}^{5}\right]\left(\frac{\sigma_{\mathrm{osc}}^{\prime}}{\sigma_{\mathrm{osc}}}\right)^{3}\left(\delta \sigma_{*}\right)^{3}
\end{aligned}
$$

$\#^{2}$ In the following, we do not consider isocurvature fluctuations. For studies of isocurvature fluctuations in the curvaton model, see e.g. [40 44]. 
where $r_{\text {dec }}$ is defined as

$$
\left.r_{\mathrm{dec}} \equiv \frac{3 \rho_{\sigma}}{4 \rho_{\mathrm{rad}}+3 \rho_{\sigma}}\right|_{\text {decay }} .
$$

We assume that, for a given inflationary Hubble parameter $H_{*}$, the curvaton decay rate can be adjusted such that the correct perturbation amplitude $\zeta \simeq 10^{-5}$ can be obtained.

The non-linearity parameter $f_{\mathrm{NL}}$ is then given by $\# 3$

$$
f_{\mathrm{NL}}=\frac{5}{4 r_{\mathrm{dec}}}\left(1+\frac{\sigma_{\mathrm{osc}} \sigma_{\mathrm{osc}}^{\prime \prime}}{\sigma_{\mathrm{osc}}^{\prime 2}}\right)-\frac{5}{3}-\frac{5 r_{\mathrm{dec}}}{6} .
$$

Let us now discuss the scale dependence of $f_{\mathrm{NL}}$ in the self-interacting curvaton model. The scale dependence of $f_{\mathrm{NL}}$ is defined as (see [33] for details)

$$
n_{f_{\mathrm{NL}}} \equiv \frac{d \ln \left|f_{\mathrm{NL}}\right|}{d \ln k}
$$

where we regard $n_{f_{\mathrm{NL}}}$ as the spectral index for $f_{\mathrm{NL}}$. From this definition, treating $n_{f_{\mathrm{NL}}}$ as a constant is equivalent to assuming the power-law form:

$$
f_{\mathrm{NL}} \propto k^{n_{f_{\mathrm{NL}}}} .
$$

For the self-interacting curvaton model, the spectral index can be calculated as [33] (for a very recent, alternative method of calculation see [34])

$$
\begin{aligned}
n_{f_{\mathrm{NL}}} & =\frac{V^{\prime \prime \prime}\left(\sigma_{*}\right)}{3 H_{*}^{2}} \frac{\sigma_{\mathrm{osc}}}{4 \sigma_{\mathrm{osc}}^{\prime}}\left[\frac{1}{4}\left(1+\frac{\sigma_{\mathrm{osc}} \sigma_{\mathrm{osc}}^{\prime \prime}}{\sigma_{\mathrm{osc}}^{\prime 2}}\right)-\frac{1}{3} r_{\mathrm{dec}}-\frac{1}{6} r_{\mathrm{dec}}^{2}\right]^{-1} \\
& =\frac{V^{\prime \prime \prime}\left(\sigma_{*}\right)}{3 H_{*}^{2}} \frac{\sigma_{\mathrm{osc}}}{\sigma_{\mathrm{osc}}^{\prime}} \frac{5}{4 r_{\mathrm{dec}} f_{\mathrm{NL}}}
\end{aligned}
$$

where a prime indicates the derivative with respect to $\sigma_{*}$, i.e., $V^{\prime \prime \prime}=d^{3} V / d \sigma^{3}$.

In addition to the spectral index $n_{f_{\mathrm{NL}}}$, the running of $n_{f_{\mathrm{NL}}}$ can also be defined analogously to the running of the spectral index of the usual spectrum as

$$
\alpha_{f_{\mathrm{NL}}} \equiv \frac{d n_{f_{\mathrm{NL}}}}{d \ln k}
$$

For the curvaton model with self-interaction, we obtain the following expression for the running $\alpha_{f_{\mathrm{NL}}} \#$ :

$$
\alpha_{f_{\mathrm{NL}}}=n_{f_{\mathrm{NL}}}\left[-\frac{V^{\prime \prime}}{3 H_{*}^{2}}+2 \epsilon-\frac{V^{\prime \prime \prime \prime}}{3 H_{*}^{2}} \frac{V^{\prime}}{V^{\prime \prime \prime}}\right]-n_{f_{\mathrm{NL}}}^{2} .
$$

\footnotetext{
\#3 For the definition and details of the calculations of the non-linearity parameters in the model, we refer the reader to e.g., [21].

\#4 We thank Qing-Guo Huang for pointing out an error in this formula in previous versions of this paper. The result given here agrees with the arXiv version of [35].
} 
The derivative of the potential can be evaluated once $s, p, \lambda$, and the mass $m_{\sigma}$ are given. However, in the following discussion, instead of the mass we find it convenient to use the parameter $\eta_{\sigma \sigma}$, defined as the ratio between $V^{\prime \prime}$ and the Hubble parameter during inflation:

$$
\eta_{\sigma \sigma}=\frac{V^{\prime \prime}\left(\sigma_{*}\right)}{3 H_{*}^{2}}=\frac{m_{\sigma}^{2}}{3 H_{*}^{2}}\left(1+\frac{p(p-1)}{2} s\right) .
$$

Notice that the spectral index for the power spectrum in this model is given by

$$
n_{s}-1=-2 \epsilon+2 \eta_{\sigma \sigma}
$$

where $\epsilon$ is the slow-roll parameter for the inflaton sector. Thus the value of $\eta_{\sigma \sigma}$ should be small enough to be consistent with the observational constraint: $0.9358<n_{s}-1<0.9921$ (95\% C.L.) [1].

With the definition (11) for $\eta_{\sigma \sigma}$, we can express the higher derivatives of the potential as

$$
\begin{aligned}
\frac{V^{\prime \prime \prime}}{3 H_{*}^{2}} & =\eta_{\sigma \sigma} \frac{p(p-1)(p-2) s}{2+p(p-1) s} \frac{1}{\sigma_{*}}, \\
\frac{V^{\prime \prime \prime \prime}}{3 H_{*}^{2}} & =\eta_{\sigma \sigma} \frac{p(p-1)(p-2)(p-3) s}{2+p(p-1) s} \frac{1}{\sigma_{*}^{2}} .
\end{aligned}
$$

It should be noticed here that, in the self-interacting curvaton model, the value of $f_{\mathrm{NL}}$ can change its sign when parameters are varied. In fact, when $f_{\mathrm{NL}}$ changes its sign, the scale-dependence of $f_{\mathrm{NL}}$ cannot be well described by the power-law form of Eq. (7). One could also see this by noticing that the expression of $n_{f_{\mathrm{NL}}}$ given by Eq. (8) diverges when $f_{\mathrm{NL}}=0$. However, as we will discuss later, when $f_{\mathrm{NL}}$ is small enough, it would be impossible to measure the scale-dependence of $f_{\mathrm{NL}}$; for small enough $f_{\mathrm{NL}}$, it is not an observable. Furthermore, we have checked that the magnitude of the running $\alpha_{f_{\mathrm{NL}}}$ is much smaller than $n_{f_{\mathrm{NL}}}$ except in the region where $f_{\mathrm{NL}} \lesssim \mathcal{O}(1)$. In the following we do not consider the unobservable region $f_{\mathrm{NL}} \lesssim \mathcal{O}(1)$; as a consequence, for us $n_{f_{\mathrm{NL}}}$ is indeed the proper way to describe scale-dependence of $f_{\mathrm{NL}}$ in the whole range of interest.

Although in this paper we mainly focus on the scale-dependence of $f_{\mathrm{NL}}$, we can also define the scale dependence of $g_{\mathrm{NL}}$ similarly to the case of $f_{\mathrm{NL}}$ as 34 ]

$$
n_{g_{\mathrm{NL}}} \equiv \frac{d \ln \left|g_{\mathrm{NL}}\right|}{d \ln k} .
$$

Since in the curvaton model with a self-interaction term, the non-linearity parameter $g_{\mathrm{NL}}$ is given by

$$
\begin{aligned}
g_{\mathrm{NL}} & =\frac{25}{54}\left[\frac{9}{4 r_{\mathrm{dec}}^{2}}\left(\frac{\sigma_{\mathrm{osc}}^{2} \sigma_{\mathrm{osc}}^{\prime \prime \prime}}{\sigma_{\mathrm{osc}}^{\prime 3}}+3 \frac{\sigma_{\mathrm{osc}} \sigma_{\mathrm{osc}}^{\prime \prime}}{\sigma_{\mathrm{osc}}^{\prime 2}}\right)-\frac{9}{r_{\mathrm{dec}}}\left(1+\frac{\sigma_{\mathrm{osc}} \sigma_{\mathrm{osc}}^{\prime \prime}}{\sigma_{\mathrm{osc}}^{\prime 2}}\right)\right. \\
& \left.+\frac{1}{2}\left(1-9 \frac{\sigma_{\mathrm{osc}} \sigma_{\mathrm{osc}}^{\prime \prime}}{\sigma_{\mathrm{osc}}^{\prime \prime}}\right)+10 r_{\mathrm{dec}}+3 r_{\mathrm{dec}}^{2}\right]
\end{aligned}
$$


as in the case for $n_{f_{\mathrm{NL}}}$, a similar calculation (consistent with the results of [34]) yields $n_{g_{\mathrm{NL}}}$ :

$$
\begin{aligned}
& n_{g_{\mathrm{NL}}}=\left[\frac{9}{4}\left(\frac{V^{\prime \prime \prime \prime}\left(\sigma_{*}\right)}{3 H_{*}^{2}}-3 \eta_{\sigma \sigma}^{2}\right)\left(\frac{\sigma_{\mathrm{osc}}}{\sigma_{\mathrm{osc}}^{\prime}}\right)^{2}+\frac{27}{4}\left(\frac{V^{\prime \prime \prime}\left(\sigma_{*}\right)}{3 H_{*}^{2}}\right)\left\{\frac{\sigma_{\mathrm{osc}}^{2} \sigma_{\mathrm{osc}}^{\prime \prime}}{\left(\sigma_{\mathrm{osc}}^{\prime}\right)^{3}}+\left(1-\frac{4}{3} r_{\mathrm{dec}}-\frac{2}{3} r_{\mathrm{dec}}^{2}\right) \frac{\sigma_{\mathrm{osc}}}{\sigma_{\mathrm{osc}}^{\prime}}\right\}\right] \\
& \times\left[\frac{9}{4}\left(\frac{\sigma_{\mathrm{osc}}^{2} \sigma_{\mathrm{osc}}^{\prime \prime \prime}}{\sigma_{\mathrm{osc}}^{\prime 3}}+3 \frac{\sigma_{\mathrm{osc}} \sigma_{\mathrm{osc}}^{\prime \prime}}{\sigma_{\mathrm{osc}}^{\prime 2}}\right)-9 r_{\mathrm{dec}}\left(1+\frac{\sigma_{\mathrm{osc}} \sigma_{\mathrm{osc}}^{\prime \prime}}{\sigma_{\mathrm{osc}}^{\prime 2}}\right)+\frac{r_{\mathrm{dec}}^{2}}{2}\left(1-9 \frac{\sigma_{\mathrm{osc}} \sigma_{\mathrm{osc}}^{\prime \prime}}{\sigma_{\mathrm{osc}}^{\prime 2}}\right)+10 r_{\mathrm{dec}}^{3}+3 r_{\mathrm{dec}}^{4}\right]^{-1} .
\end{aligned}
$$

\section{$3 \quad n_{f_{\mathrm{NL}}}$ in the curvaton model}

We have scanned the parameter space and evaluated the spectral index $n_{f_{\mathrm{NL}}}$ numerically. In the left panel of Fig. 1, we plot the value of $n_{f_{\mathrm{NL}}}$ as a function of the self-interaction power $p$. In the figure, we have chosen the parameters as $s=0.05$ (red solid line), 0.07 (green dashed line) and for the rest of this paper we choose

$$
\eta_{\sigma \sigma}=0.005 \text {. }
$$

Note that the scale dependence of both $n_{f_{\mathrm{NL}}}$ and $n_{g_{\mathrm{NL}}}$ is proportional to $\eta_{\sigma \sigma}$, so they would both be larger if we chose a larger $\eta_{\sigma \sigma}$. The corresponding values of $f_{\mathrm{NL}}$ are also shown in the right panel. We have chosen the value of $r_{\text {dec }}$ such that we obtain $f_{\mathrm{NL}}=50$ at $p=6$ for each relative self-interaction strength $s$ (therefore the values of $r_{\text {dec }}$ are different for each case). As discussed in the previous section, when $f_{\mathrm{NL}}$ very small, with $f_{\mathrm{NL}} \lesssim \mathcal{O}(1)$, the power-law form cannot well describe the scale dependence of $f_{\mathrm{NL}}$. To emphasize this fact we cut out the regions with $f_{\mathrm{NL}} \leq 10$.

According to [30], future observations such as CMBpol may be able to detect the scale dependence of $f_{\mathrm{NL}}$. The observational error has been estimated as (for the fiducial value of $\left.n_{f_{\mathrm{NL}}}=0\right)$

$$
\Delta n_{f_{\mathrm{NL}}} \simeq 0.05 \frac{50}{f_{\mathrm{NL}}} \frac{1}{\sqrt{f_{\mathrm{sky}}}},
$$

where $f_{\text {sky }}$ is the fraction of the sky measured. Hence in the parameter space where the theoretical estimate $n_{f_{\mathrm{NL}}} \geq \Delta n_{f_{\mathrm{NL}}}$ we may be able to constrain the self-interacting curvaton model by the scale dependence of $f_{\mathrm{NL}}$. For example, as can be read off from figure 2, when $p=6$ and $s=0.07$, the spectral index is $n_{f_{\mathrm{NL}}} \simeq 0.1$, which should be observable with future observations.

To chart the parameter space where $n_{f_{\mathrm{NL}}}$ is a useful tool for model building, we plot in Fig. 2 the contours of $n_{f_{\mathrm{NL}}}$ and $f_{\mathrm{NL}}$ in the $s-r_{\text {dec }}$ plane for the cases of $p=6$ and 8 . From the expression of Eq. (5), one can see that while generally $f_{\mathrm{NL}}$ is proportional to $1 / r_{\text {dec }}$, in the self-interacting curvaton model $f_{\mathrm{NL}}$ also depends on $s$ through the non-linear evolution of the curvaton field. This is encoded in $\sigma_{\mathrm{osc}}$ and its derivatives. On the other hand, when $r_{\text {dec }}$ is small, $n_{f_{\mathrm{NL}}}$ does not depend on $r_{\mathrm{dec}}$ but strongly depends on $s$. Since the scale-dependence of $f_{\mathrm{NL}}$ mainly originates from the non-linear evolution of $\sigma$ due to 

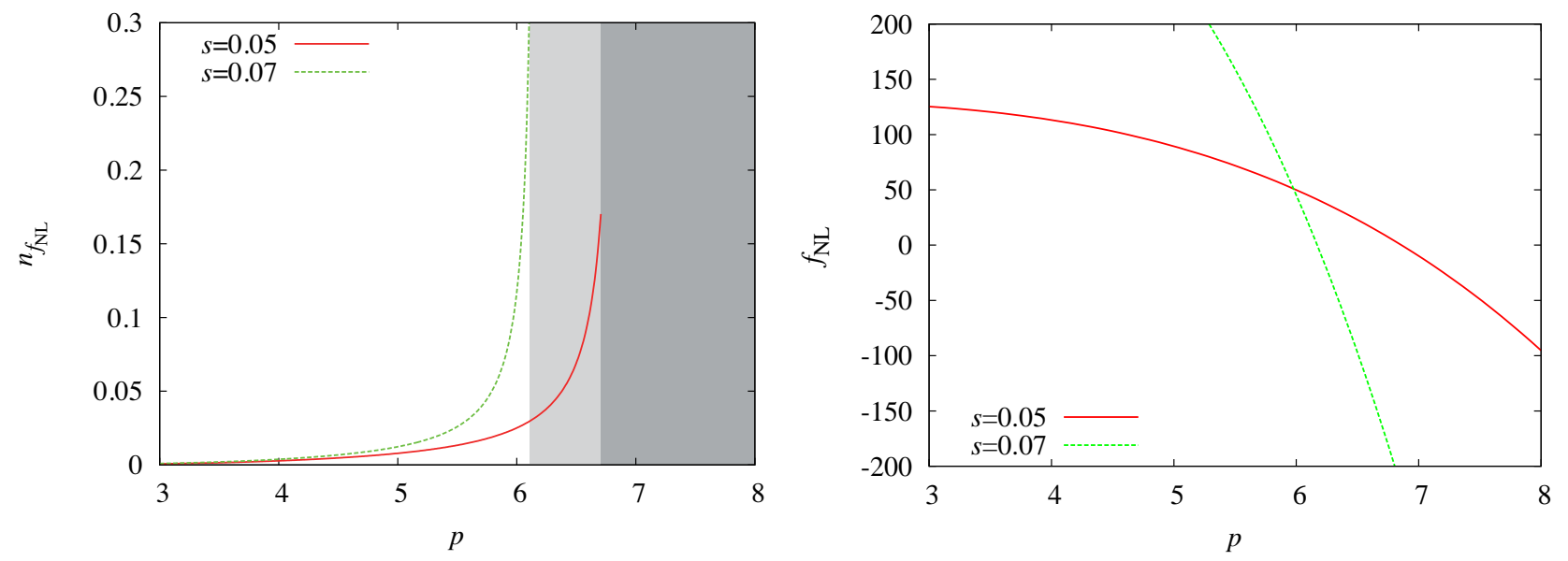

Figure 1: Plots of $n_{f_{\mathrm{NL}}}$ (left panel) and $f_{\mathrm{NL}}$ (right panel) as a function of the power $p$. Here we take $s=0.05$ (red line) and 0.07 (green line) with $\eta_{\sigma \sigma}=0.005$. The shaded areas correspond to the regions where the power-law form $f_{\mathrm{NL}} \propto k^{n_{f_{\mathrm{NL}}}}$ does not hold.
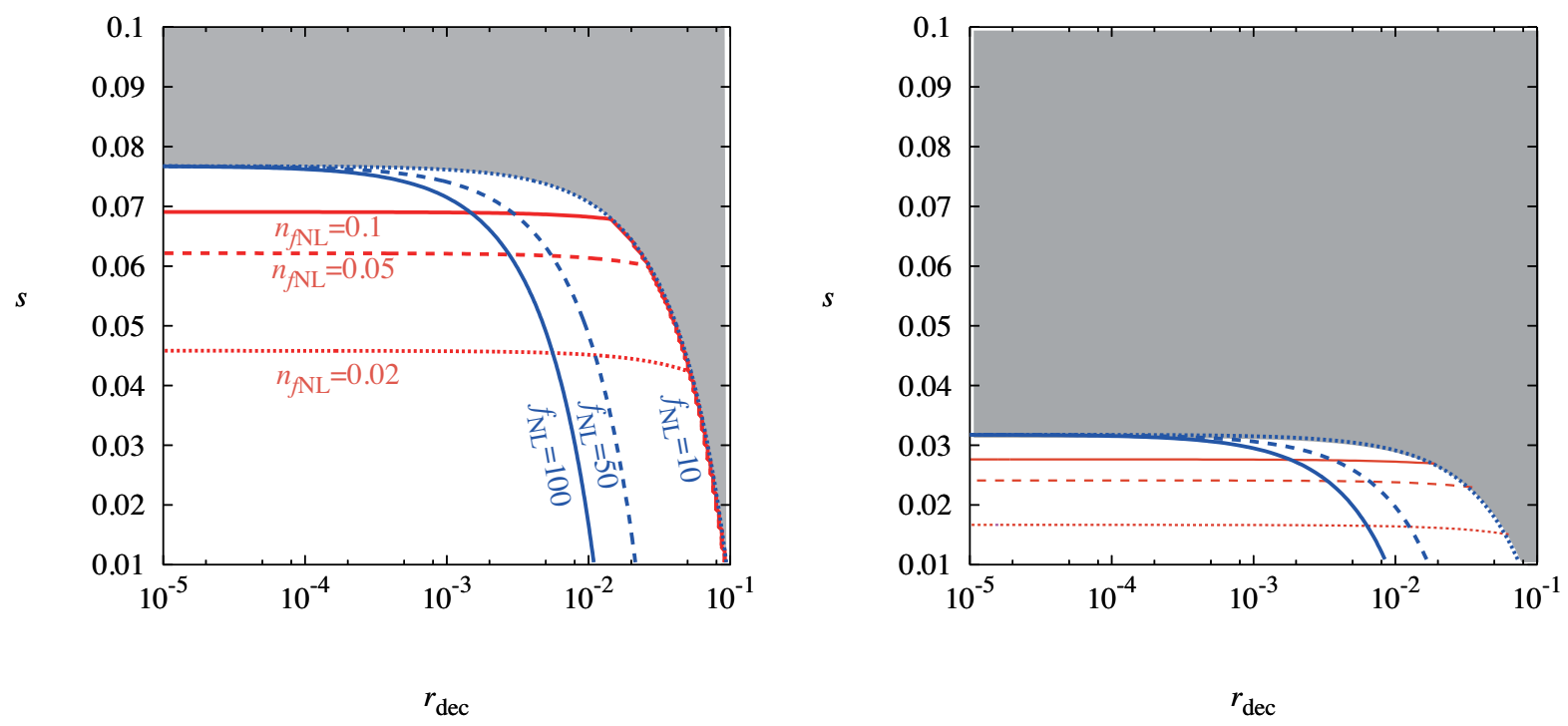

Figure 2: Contours of $f_{\mathrm{NL}}$ and $n_{f_{\mathrm{NL}}}$ for $p=6$ (left panel) and 8 (right panel). The region with $f_{\mathrm{NL}}<10$ is shaded. 


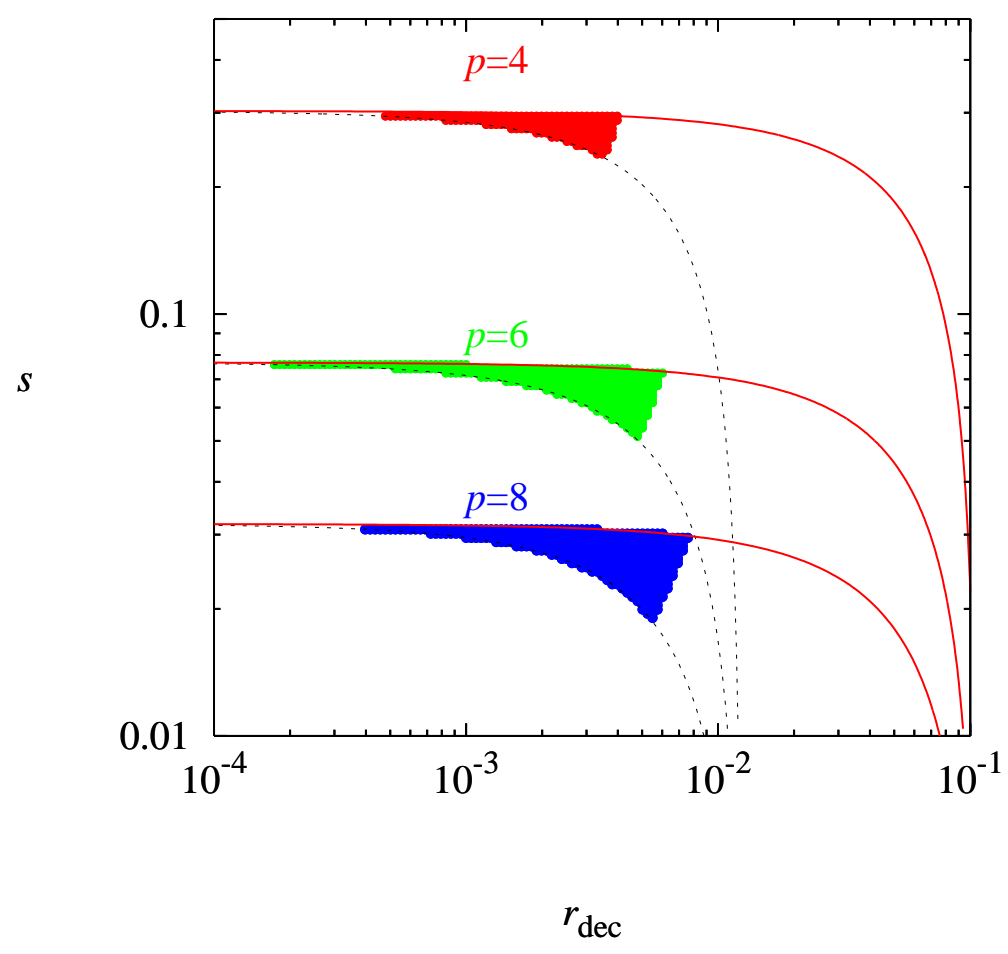

Figure 3: Regions testable in future observations. Shown is the parameter space where the theoretical prediction for $n_{f_{\mathrm{NL}}}$ exceeds $\Delta n_{f_{\mathrm{NL}}}$ given in Eq. (19), (i.e., region where $n_{f_{\mathrm{NL}}}>\Delta n_{f_{\mathrm{NL}}}=0.05 \times\left(50 / f_{\mathrm{NL}}\right)$ is satisfied $)$, for $10 \leq f_{\mathrm{NL}} \leq 100$. 
the higher order term in the potential, the value of $s$ controls $n_{f_{\mathrm{NL}}}$. Notice that, when $r_{\text {dec }} \ll \mathcal{O}(1)$, the sign of $f_{\mathrm{NL}}$ is determined by the factor $1+\sigma_{\mathrm{osc}} \sigma_{\text {osc }}^{\prime \prime} / \sigma_{\text {osc }}^{\prime 2}$ which appears in the first term in Eq. (5). This factor depends on $s$ and $p$, but not on $r_{\text {dec }}$. This is the reason why the contours of $f_{\mathrm{NL}}$ in Fig. 2 appear to converge at $s \simeq 0.077$ and 0.032 for $p=6$ and 8 , respectively, where $f_{\mathrm{NL}}$ abruptly switches from a positive value to a negative one. The region around this line also corresponds to the breakdown of the power-law description for the scale-dependence. By cutting out the region with $f_{\mathrm{NL}}<10$, which anyway is irrelevant from an observational viewpoint, we therefore guarantee that the power-law description is valid.

Since the sensitivity of $n_{f_{\mathrm{NL}}}$ in future observations depends on the value of $f_{\mathrm{NL}}$, as indicated in Eq. (19), the self-interacting curvaton model may be tested in certain regions of the parameter space. This is depicted in Fig. 3, where the regions with $n_{f_{\mathrm{NL}}} \geq \Delta n_{f_{\mathrm{NL}}}$ are shown for the cases $p=4,6$ and 8 . Here we conservatively adopt the range $10<f_{\mathrm{NL}}<100$. If future observations find a non-zero value for $n_{f_{\mathrm{NL}}}$, the self-interacting curvaton model must lie inside the colored regions. On the other hand, if no scale-dependence of $f_{\mathrm{NL}}$ will be detected, the colored region will be excluded. Planck is expected to have about half of the sensitivity to $n_{f_{\mathrm{NL}}}$ compared to (19), but large scale structure probes may become even more sensitive to $n_{f_{\mathrm{NL}}}$ than CMBpol.

\section{Constraints implied by the trispectrum: $g_{\mathrm{NL}}$}
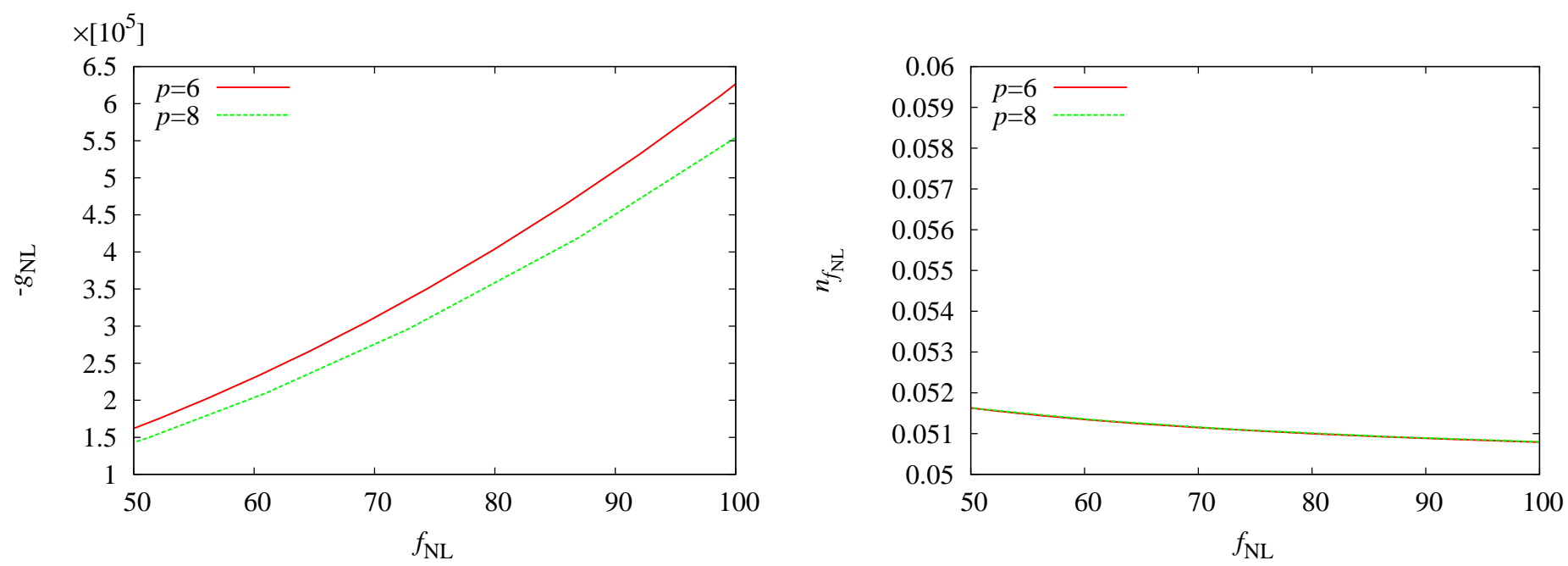

Figure 4: Plots of $n_{f_{\mathrm{NL}}}$ (right) and $g_{\mathrm{NL}}$ (left) for the cases with $(p, s)=(6,0.0622)$ and $(8,0.0214)$. The value of $s$ is chosen such that the cases of $p=6$ and 8 give almost the same values of $n_{f_{\mathrm{NL}}}$ as a function of $f_{\mathrm{NL}}$. 


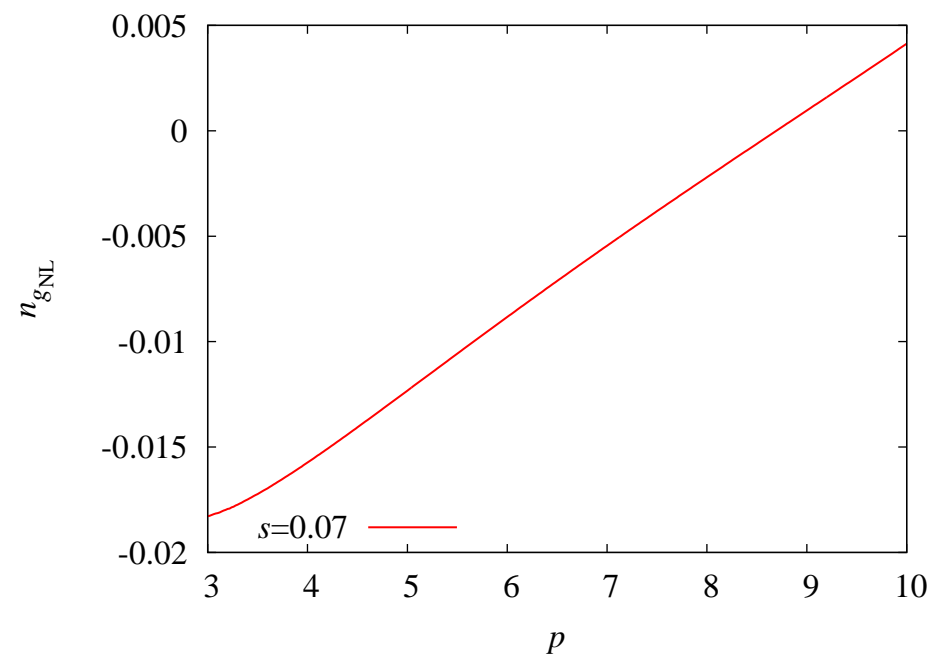

Figure 5: Plot of $n_{g_{\mathrm{NL}}}$ as a function of the power $p$ for $s=0.07$.

In the previous section, we have shown that $n_{f_{\mathrm{NL}}}$ can be a useful tool to probe/test the self-interacting curvaton model. However, even if we can observe $n_{f_{\mathrm{NL}}}$ and $f_{\mathrm{NL}}$, the situation remains degenerate: different combinations of the power $p$, the strength $s$ and $r_{\mathrm{dec}}$ can give the same values of $f_{\mathrm{NL}}$ and $n_{f_{\mathrm{NL}}}$. To demonstrate this, in the right panel of Fig. 4, we plot $n_{f_{\mathrm{NL}}}$ as a function of $f_{\mathrm{NL}}$, which is almost equivalent to the plot as a function of $r_{\mathrm{dec}}$, for the cases of $p=6$ and 8. To give an example, by fixing $s=0.0622$ and 0.0214 for $p=6$ and 8 , respectively, the lines for $p=6$ and 8 almost coincide, as is shown in the right panel of Fig. 4. However, the predictions for $g_{\mathrm{NL}}$ are in general different. This is demonstrated in the left panel of Fig. 4, where we show $g_{\mathrm{NL}}$ as a function of $f_{\mathrm{NL}}$ for the same parameters as in the right panel. This figure indicates that, in principle, it is possible break the degeneracy between $n_{f_{\mathrm{NL}}}$ and $f_{\mathrm{NL}}$ by including $g_{\mathrm{NL}}$ in the analysis. Thus combining together information about the bispectrum amplitude, the spectral index of the bispectrum, and the amplitude of the trispectrum, one may actually fix completely the parameters of the simplest self-interacting curvaton model (1). We note that $\tau_{\mathrm{NL}}$ in this model does not give new information, since it satisfies $\tau_{\mathrm{NL}}=\left(6 f_{\mathrm{NL}} / 5\right)^{2}$ (for more discussion on the trispectrum see [45, 46]), but testing this consistency relation is still valuable since it can determine whether it was justified to neglect the the inflaton field fluctuations [19].

Furthermore, we can also address the scale-dependence of $g_{\mathrm{NL}}$; the appropriate expression was already given in Sec. 2. In Fig. 5, $n_{g_{\mathrm{NL}}}$ is plotted as a function of the power $p$ for $s=0.07$. It is worth noticing that $n_{g_{\mathrm{NL}}}$ becomes larger the smaller $p$ is. This is in contrast to the behavior of $n_{f_{\mathrm{NL}}}$, which becomes smaller as the value of the power $p$ becomes smaller, as is shown in Fig. 1. Thus, in future observations it may be possible to see the scale-dependence of $g_{\mathrm{NL}}$ even if we cannot detect the scale-dependence of $f_{\mathrm{NL}}$. 
Hence, in addition to $g_{\mathrm{NL}}$ itself, the scale-dependence of $g_{\mathrm{NL}}$ might also provide a tool to probe/test the self-interacting curvaton model.

\section{Conclusion and discussion}

In this paper, we have discussed the scale dependence of the non-linear parameters such as $f_{\mathrm{NL}}$ and $g_{\mathrm{NL}}$ in a curvaton model with a simple self-interaction term given by the potential (11). The free parameters of the model are the curvaton mass $m$, the power of the self-interaction term $p$, and its relative strength $s$. The complete parametrization of the curvature perturbation would require also the knowledge of $H_{*}$, determined by the inflaton sector. In principle $H_{*}$ can be observationally fixed by a detection of primordial gravitational waves, but their amplitude is likely to be very small in the curvaton scenario. In a complete model, the curvaton decay rate might not be a totally independent parameter, but here we assume that, for a given $H_{*}$, the decay rate can be tuned to yield the correct perturbation amplitude $\zeta \simeq 10^{-5}$. In such a situation, as has been argued before, even a modest amount of non-linearity in the curvaton potential [15, 21] may lead to observable consequences. Therefore, although $f_{\mathrm{NL}}$ is scale-invariant when the curvaton potential is quadratic, in many cases the spectral index $n_{f_{\mathrm{NL}}}$ of the bispectrum can be detectable when one includes the self-interaction term; this fact is depicted in Fig. 1.

Since the observational sensitivity to the scale-dependence of $f_{\mathrm{NL}}$ depends on the magnitude of $f_{\mathrm{NL}}$, there are regions where the self-interacting curvaton model parameters can be pinpointed by the observations of $f_{\mathrm{NL}}$ and its scale dependence $n_{f_{\mathrm{NL}}}$. The testable domains in the parameter space are shown in Fig. 3. If no scale-dependence of $f_{\mathrm{NL}}$ will be found, these regions would be excluded so that for the self-interacting curvaton model, the scale-dependence can indeed provide us with clear-cut evidence for or against the model.

In addition to the scale-dependence of $f_{\mathrm{NL}}$, we also studied the non-linearity parameter of the trispectrum, $g_{\mathrm{NL}}$, and its scale-dependence $n_{g_{\mathrm{NL}}}$. As is shown in Fig. 4, even though in principle there is a degeneracy in the space of model parameters in that a given pair of values for $n_{f_{\mathrm{NL}}}$ and $f_{\mathrm{NL}}$ is produced by several combinations of $(p, s)$, the degeneracy can be broken by $g_{\mathrm{NL}}$. The spectral index of $g_{\mathrm{NL}}$ could be used to probe the scale-dependence of non-Gaussianity in the self-interacting curvaton model at least for some of the cases where $n_{f_{\mathrm{NL}}}$ is not observable.

So far the studies of non-Gaussianity in the curvaton and other models have focused

on the non-linearity parameters $f_{\mathrm{NL}}, \tau_{\mathrm{NL}}$ and $g_{\mathrm{NL}}$. Here we have pointed out that the scale dependence could also provide a realistic and useful tool to test the models. In particular, we have argued that the self-interacting curvaton model parameters could actually be completely fixed by the combined observations of $f_{\mathrm{NL}}, g_{\mathrm{NL}}$, and the spectral index $n_{f_{\mathrm{NL}}}$. The fact that the projected observational sensitivity of the latter appears in many cases to be much higher than would be required for verification of the theoretical estimates is of course encouraging. It also lends some hope for the expectation that the physical origin of the primordial perturbation can be determined in the not too distant future. 
Acknowledgments: We thank Sami Nurmi for useful comments on the draft. We also thank Qing-Guo Huang for pointing out an error in Eq. (10). T.T. would like to thank the Helsinki Institute of Physics for the hospitality during the visit, where a part of this work has been done. This work is supported in part the Grant-in-Aid for Scientific Research from the Ministry of Education, Science, Sports, and Culture of Japan No. 19740145 (T.T.), and in part by the Academy of Finland grants 218322 and 131454 (K.E.). The authors also wish to thank the organizers of the workshops "The non-Gaussian universe" (YITP-T-0905) and "Cosmology - The Next Generation" (YKIS2010 symposium), both at the Yukawa Institute for Theoretical Physics, Kyoto, Japan, for hospitality, where parts of this work were done.

\section{References}

[1] E. Komatsu et al., arXiv:1001.4538 [astro-ph.CO].

[2] K. Enqvist and M. S. Sloth, Nucl. Phys. B 626, 395 (2002) arXiv:hep-ph/0109214;

[3] D. H. Lyth and D. Wands, Phys. Lett. B 524, 5 (2002) arXiv:hep-ph/0110002;;

[4] T. Moroi and T. Takahashi, Phys. Lett. B 522, 215 (2001) [Erratum-ibid. B 539, 303 (2002)] arXiv:hep-ph/0110096.

[5] V. Desjacques and U. Seljak, Phys. Rev. D 81, 023006 (2010) arXiv:0907.2257 [astro-ph.CO]].

[6] P. Vielva and J. L. Sanz, arXiv:0910.3196 [astro-ph.CO].

[7] J. Smidt, A. Amblard, A. Cooray, A. Heavens, D. Munshi and P. Serra, arXiv:1001.5026 [astro-ph.CO].

[8] N. Kogo and E. Komatsu, Phys. Rev. D 73, 083007 (2006) arXiv:astro-ph/0602099].

[9] J. Smidt, A. Amblard, C. T. Byrnes, A. Cooray, A. Heavens and D. Munshi, Phys. Rev. D 81, 123007 (2010) [arXiv:1004.1409 [astro-ph.CO]].

[10] J. M. Maldacena, JHEP 0305, 013 (2003) arXiv:astro-ph/0210603.

[11] D. H. Lyth, C. Ungarelli and D. Wands, Phys. Rev. D 67, 023503 (2003) arXiv:astro-ph/0208055.

[12] K. Dimopoulos, G. Lazarides, D. Lyth and R. Ruiz de Austri, Phys. Rev. D 68, 123515 (2003) arXiv:hep-ph/0308015.

[13] N. Bartolo, S. Matarrese and A. Riotto, Phys. Rev. D 69, 043503 (2004) arXiv:hep-ph/0309033. 
[14] D. H. Lyth and Y. Rodriguez, Phys. Rev. Lett. 95, 121302 (2005) arXiv:astro-ph/0504045.

[15] K. Enqvist and S. Nurmi, JCAP 0510, 013 (2005) arXiv:astro-ph/0508573.

[16] K. A. Malik and D. H. Lyth, JCAP 0609, 008 (2006) arXiv:astro-ph/0604387.

[17] M. Sasaki, J. Valiviita and D. Wands, Phys. Rev. D 74, 103003 (2006) arXiv:astro-ph/0607627.

[18] Q. G. Huang, arXiv:0801.0467 [hep-th].

[19] K. Ichikawa, T. Suyama, T. Takahashi and M. Yamaguchi, Phys. Rev. D 78, 023513 (2008) arXiv:0802.4138 [astro-ph]].

[20] T. Multamaki, J. Sainio and I. Vilja, Phys. Rev. D 79, 103516 (2009) arXiv:0803.2637 [astro-ph]].

[21] K. Enqvist and T. Takahashi, JCAP 0809, 012 (2008) arXiv:0807.3069 [astro-ph]].

[22] Q. G. Huang and Y. Wang, JCAP 0809, 025 (2008) arXiv:0808.1168 [hep-th]].

[23] Q. G. Huang, JCAP 0811, 005 (2008) arXiv:0808.1793 [hep-th]].

[24] M. Kawasaki, K. Nakayama and F. Takahashi, JCAP 0901, 026 (2009) arXiv:0810.1585 [hep-ph]].

[25] P. Chingangbam and Q. G. Huang, JCAP 0904, 031 (2009) arXiv:0902.2619 [astroph.CO]].

[26] K. Enqvist, S. Nurmi, G. Rigopoulos, O. Taanila and T. Takahashi, JCAP 0911, 003 (2009) arXiv:0906.3126 [astro-ph.CO]].

[27] K. Enqvist and T. Takahashi, JCAP 0912, 001 (2009) arXiv:0909.5362 [astroph.CO]].

[28] K. Enqvist, S. Nurmi, O. Taanila and T. Takahashi, JCAP 1004, 009 (2010) arXiv:0912.4657 [astro-ph.CO]].

[29] P. Chingangbam and Q. G. Huang, arXiv:1006.4006 [astro-ph.CO].

[30] E. Sefusatti, M. Liguori, A. P. S. Yadav, M. G. Jackson and E. Pajer, JCAP 0912, 022 (2009) arXiv:0906.0232 [astro-ph.CO]].

[31] C. T. Byrnes, K. Y. Choi and L. M. H. Hall, JCAP 0902, 017 (2009) arXiv:0812.0807 [astro-ph]]. 
[32] J. Kumar, L. Leblond and A. Rajaraman, JCAP 1004, 024 (2010) arXiv:0909.2040 [astro-ph.CO]].

[33] C. T. Byrnes, S. Nurmi, G. Tasinato and D. Wands, JCAP 1002, 034 (2010) arXiv:0911.2780 [astro-ph.CO]].

[34] C. T. Byrnes, M. Gerstenlauer, S. Nurmi, G. Tasinato and D. Wands, arXiv:1007.4277 [astro-ph.CO].

[35] Q. G. Huang, JCAP 1011, 026 (2010). [arXiv:1008.2641v4 [astro-ph.CO].]

[36] X. Chen, Phys. Rev. D 72, 123518 (2005) arXiv:astro-ph/0507053.

[37] M. LoVerde, A. Miller, S. Shandera and L. Verde, JCAP 0804, 014 (2008) arXiv:0711.4126 [astro-ph]].

[38] J. Khoury and F. Piazza, JCAP 0907, 026 (2009) [arXiv:0811.3633 [hep-th]].

[39] S. Renaux-Petel, JCAP 0910, 012 (2009) [arXiv:0907.2476 [hep-th]].

[40] T. Moroi and T. Takahashi, Phys. Rev. D 66, 063501 (2002) [arXiv:hep-ph/0206026].

[41] D. H. Lyth and D. Wands, Phys. Rev. D 68, 103516 (2003) arXiv:astro-ph/0306500.

[42] M. Beltran, Phys. Rev. D 78, 023530 (2008) arXiv:0804.1097 [astro-ph]].

[43] T. Moroi and T. Takahashi, Phys. Lett. B 671, 339 (2009) arXiv:0810.0189 [hep$\mathrm{ph}]$.

[44] T. Takahashi, M. Yamaguchi and S. Yokoyama, Phys. Rev. D 80, 063524 (2009) arXiv:0907.3052 [astro-ph.CO]].

[45] D. Seery and J. E. Lidsey, JCAP 0701, 008 (2007) arXiv:astro-ph/0611034.

[46] C. T. Byrnes, M. Sasaki and D. Wands, Phys. Rev. D 74, 123519 (2006) arXiv:astro-ph/0611075. 